\title{
Topics and techniques in clinical supervision in psychotherapy training
}

\author{
Florian Weck ${ }^{1 *}$, Yvonne M. Kaufmann ${ }^{1,2}$ and Michael Witthöft ${ }^{2}$ \\ ${ }^{1}$ Department of Clinical Psychology and Psychotherapy, University of Potsdam, Potsdam, Germany \\ ${ }^{2}$ Department of Clinical Psychology, Psychotherapy and Experimental Psychopathology, University of \\ Mainz, Mainz, Germany
}

Received 27 January 2017; Accepted 7 June 2017

\begin{abstract}
Clinical supervision is regarded as one of the most important components of psychotherapy training. In clinical practice, it has been found that the implementation of clinical supervision varies substantially and often differs from the recommendations made in the literature. The objective of the current study was to investigate the frequency of topics (e.g. ethical issues) and techniques (e.g. role play) in the clinical supervision of psychotherapy trainees in Germany. To this end, we considered supervisions in cognitive behavioural therapy (CBT) and psychodynamic therapy (PT). A total of 791 psychotherapy trainees (533 CBT and 242 PT) were asked via the internet to provide information about their current supervision sessions. We found that clinical supervision in psychotherapy training addressed topics that are central for the effective treatment of supervised patients (i.e. therapeutic interventions, therapeutic alliance, maintaining factors, and therapeutic goals). However, the most frequently used intervention in clinical supervision in psychotherapy training was case discussion. Rarely were techniques used that allowed the supervisor to give the supervisee feedback based on the supervisee's demonstrated competencies. For example, $46 \%$ of the supervisors never used audiotapes or videotapes in the supervision. Differences between CBT and PT were rather small. Current practice regarding the techniques used in clinical supervision for psychotherapy trainees contradicts recommendations for active and feedback-oriented clinical supervision. Thus the potential of clinical supervision might not be fully used in clinical practice.
\end{abstract}

Key words: clinical supervision, psychotherapy trainees, psychotherapy training, supervisory strategies, therapeutic competence

\section{Introduction}

One empirically derived definition of clinical supervision is provided by Milne (2007, p. 440), who defines clinical supervision as 'the formal provision by senior/qualified health practitioners of an intensive relationship-based education and training that is case-focused

\footnotetext{
*Author for correspondence: Dr Florian Weck, Department of Clinical Psychology and Psychotherapy, University of Potsdam, Karl-Liebknecht-Straße 24-25, D-14476 Potsdam, Germany. E-mail: fweck@ uni-potsdam.de
} 
and which supports, directs and guides the work of colleagues (supervisees)'. The functions of clinical supervision are quality control, the maintenance and facilitation of supervisees' competence and capability, and helping supervisees to work effectively. This definition and these functions imply that clinical supervision should play an important role in psychotherapy training. Thus it is not surprising that clinical supervision represents a necessary element in psychotherapy training in many countries (Strauß and Kohl, 2009). Psychotherapy trainees evaluate clinical supervision as one of the most important and useful parts of their training (Nodop et al., 2010). The important role of clinical supervision is supported by empirical research indicating that clinical supervision has positive effects on supervisees' therapeutic development and competencies (e.g. Lambert and Ogles, 1997; Wheeler and Richards, 2007; Rakovshik and McManus, 2010; Bambling, 2014).

The teaching process in clinical supervision can be heterogeneous, and clinical supervision may address various topics. For example, the psychotherapeutic alliance or the patient's homework may be discussed. Moreover, these topics may be addressed by different techniques (e.g. giving information, conducting a role play) to promote the supervisee's learning. Models that describe the development of therapeutic competence (Milne et al., 2001; Sharpless and Barber, 2009) consider qualitatively different competence stages (e.g. novice, advance beginner, expert), which are associated with different learning needs. Therefore, clinical supervision should be adapted to the different learning stages of the supervisees and should therefore differ between accredited therapists and psychotherapy trainees in different stages of their training.

Recommendations regarding clinical supervision emphasize the importance of direct observation (live or videotaped) of the demonstrated competencies to provide accurate feedback (Falender and Shafranske, 2014). Feedback might be especially important in clinical supervision because psychotherapists have difficulty adequately evaluating their own therapeutic competencies (e.g. Martino et al., 2009; Walfish et al., 2012; Weck et al., 2015). In particular, in psychotherapy training, the need for accurate self-evaluation of competencies is essential for the development of psychotherapeutic competencies. Therefore, it has been recommended that the supervisor's direct observation of the supervisee's therapy sessions and feedback regarding therapeutic work should be a central element of clinical supervision in psychotherapy training (O'Donovan et al., 2011).

The importance of the use of feedback, active learning approaches, and structuring techniques in clinical supervision was also confirmed by a systematic review (Milne et al., 2010). That review examined 24 studies in which clinical supervision demonstrated a positive impact on supervisees' learning in cognitive behavioural therapy (CBT). The most common strategies of effective supervision were feedback (in nearly $80 \%$ of the studies), followed by the use of audiotapes or videotapes, multimodal methods of teaching (e.g. live demonstration, role play rehearsal), and prioritization (e.g. discussion of agenda).

Psychotherapy research that compares different supervision approaches also shows that feedback that is based on direct observations is particularly important to improve therapeutic competencies. Thus live supervision, which allows direct insight in the therapeutic process and immediate supervisory interventions, has been found to be superior to less processoriented approaches (Smith et al., 2012; Carmel et al., 2016; Weck et al., 2016). In addition, training methods that provide video-based feedback regarding demonstrated therapeutic competencies have been shown to be effective in improving therapeutic competencies (Miller et al., 2004; Weck et al., 2017). 
Psychodynamically oriented supervisory models emphasize the similarity between psychotherapy and clinical supervision. Thus a competent psychotherapist is also considered a competent supervisor (Strauß et al., 2010). For example, in psychodynamic treatments, structure has another importance than in CBT (Cutler et al., 2004). Therefore, in contrast to CBT-oriented supervision, structuring and active supervision techniques are less relevant in clinical supervision than working out the insight and understanding of the psychodynamic process using psychodynamic methods.

Little is known about the exact type and frequency of the use of different supervision strategies in clinical practice. One exception is the study by Townend et al. (2002), who investigated the strategies utilized in clinical supervision. In that study, 170 CBT psychotherapists in the United Kingdom (UK) [accredited by the British Association of Behavioural and Cognitive Psychotherapy (BABCP)] described the practices involved in their supervision. Participants had to evaluate whether 16 specific topics (e.g. ethical issues) and eight specific techniques (e.g. homework tasks) were used 'never', 'rarely', 'sometimes' or 'often' in their supervision sessions. Based on the findings, the authors concluded that overall, clinical supervision was found to be less structured and active than psychotherapy. Strategies that enable the supervisee to receive feedback regarding his/her demonstrated competencies, such as the consideration of audiotapes or videotapes, were seldom used (approximately 52\% of the trainees reported that audiotapes or videotapes were 'never' used). Role play was also seldom used (approximately $81 \%$ of the trainees reported that role plays were 'rarely' or 'never' used). Case discussions were the most frequently used supervision technique (approximately $90 \%$ of the trainees reported that case discussions were used 'often'). It is remarkable that half of the therapists reported that strategies that allow supervisors to give feedback regarding demonstrated competencies and that were considered particularly important for the development of therapeutic competencies were not used.

Two recent investigations complement the study by Townend et al. (2002) by considering the supervisors' perspectives (Reiser and Milne, 2016; Gonsalvez et al., 2017). In the study by Gonsalvez et al. (2017), 113 accredited supervisors of postgraduate psychology trainees in Australia were questioned regarding practices in clinical supervision. The study addressed core supervisor competences, i.e. goal-setting, providing formative feedback, and conducting summative assessments. Active supervision strategies were used more often than in the study by Townend et al. (2002). For example, only $22 \%$ of the supervisors reported 'never' or 'rarely' using direct observations of the supervisee in sessions. In addition, the review of audiotaped or videotaped recordings of sessions was 'never' used in only $29 \%$ of the cases (in the study by Townend et al. (2002), 52\% of the respondents 'never' reviewed audio or video recordings). However, it is still of significant concern and against recommendations that only one-third of the cases never reviewed audio or videotapes.

In the study by Reiser and Milne (2016), 110 supervisors and trainers in the UK (accredited by the BABCP) were asked which methods they used in clinical supervision. In accordance with the study by Townend et al. (2002), case discussion was the most often used strategy in clinical supervision. However, supervisors reported using active strategies (e.g. role play, reviewing audio/video recordings) and structuring strategies (e.g. agenda setting) more frequently in clinical supervision than in the study by Townend et al. (2002). For example, $100 \%$ of the supervisors used role play and $99 \%$ of the supervisors used audio or video recordings 'sometimes' or 'frequently'. The difference in these findings might be explained by the different perspectives (i.e. supervisor versus supervisee), different samples 
(the supervisors in the study of Reiser and Milne occupied leadership roles within CBT supervision), or by a relevant and desirable change in the supervisory practice.

The study by Townend et al. (2002) examined supervisory practice in CBT in the UK more than a decade ago, raising the question of whether this supervisory practice has changed and whether this practice is different in other countries. The studies of Gonsalvez et al. (2017) in Australia and Reiser and Milne (2016) in the UK showed that supervisory practice might have changed towards the use of more active and structuring methods; however, these studies are difficult to compare because their information is based on different perspectives (i.e. supervisor versus supervisee).

In Germany, clinical supervision is a compulsory part of state-approved psychotherapy training. Psychotherapy training includes different training modules, one of which includes 150 hours of supervision. Thus clinical supervision is of high importance in German psychotherapy training, which is associated with high financial costs within psychotherapy training (Weck, 2013). In Germany, CBT and psychodynamic therapy (PT, i.e. psychodynamic psychotherapy and psychoanalysis) are part of state-approved patient care. However, there is no formal state-approved training programme for clinical supervisors in Germany. The requirements for licensure as a supervisor in psychotherapy training are one's own clinical practice (five years after accreditation as a psychotherapist) and activities as a lecturer in psychotherapy training (for at least three years). Therefore, it is unclear how familiar supervisors in Germany are with the current recommendations for supervisory practice.

The aim of the present study was to investigate topics and techniques of clinical supervision currently practised in state-approved psychotherapy training in Germany. The current study is therefore a replication of the study by Townend et al. (2002) in Germany. Moreover, the current study extends the study by Townend et al. (2002) because we considered psychotherapy trainees and supervision in CBT as well as in PT. Because of the different supervisory models in CBT and PT, strong differences between supervisory practices in these different therapeutic orientations can be expected. Therefore, we hypothesized a stronger focus on intervention-related topics in CBT supervisions and a stronger focus on the relationship between the patient and therapist in PT supervisions (hypothesis 1). Furthermore, we hypothesized that PT supervisions would be less structured and less active than CBT supervisions (hypothesis 2). Moreover, we expected that the supervisees' progress in psychotherapy training (i.e. received supervision sessions, duration of psychotherapy training) would influence the frequency of supervisory topics and techniques (hypothesis 3 ).

\section{Method}

\section{Setting and design}

The current study investigated clinical supervision for psychotherapy trainees in Germany. In Germany, health insurance funds pay for psychotherapeutic treatment. Thus CBT and PT (i.e. psychodynamic psychotherapy and psychoanalysis) are state approved. The law regulates the licensure and psychotherapy training of these approaches. The requirement for psychotherapy training is a master's degree in psychology, medicine, or educational science. The training programme lasts at least three years, including a minimum of 4200 hours of different content [e.g. workshops (minimum of 600 hours), supervision (minimum of 120 hours), and psychotherapeutic treatment of patients (minimum of 600 hours)], and concludes with an 


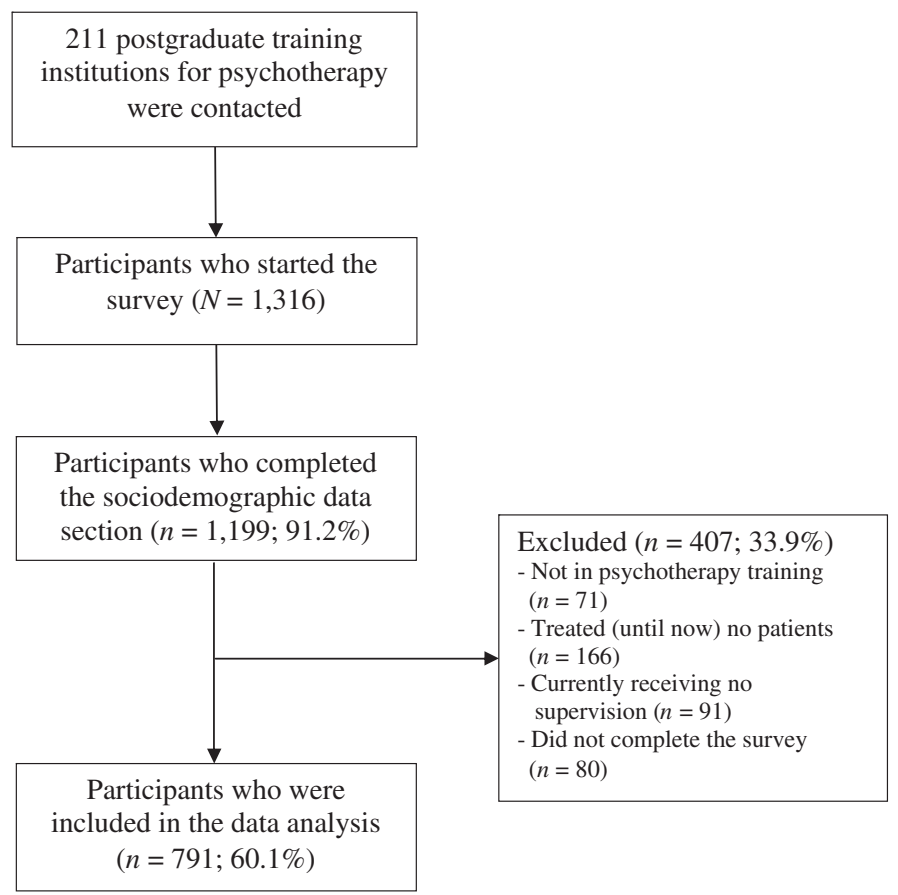

Fig. 1. Participant flowchart

oral and written state examination. The supervision of treated patients is thus an important and necessary part of psychotherapy training. At least every fourth therapy session must be supervised by a licensed supervisor.

The current study was conducted between November 2015 and January 2016. Participants were recruited in postgraduate psychotherapy training institutions (a total of 211 institutions in Germany). We contacted the institutions by email and asked them to forward a link to the online questionnaire to their trainees. The inclusion criteria of the current study were as follows: (a) participating in psychotherapy training, (b) currently treating at least one patient, (c) receiving clinical supervision, and (d) informed consent. Figure 1 shows the participants' flowchart.

Because the psychoanalyses group $(n=72)$ was relatively small and because of the conceptual closeness to psychodynamic psychotherapy $(n=163)$, both groups were pooled into one group with the trainees who were trained in psychodynamic psychotherapy and psychoanalysis $(n=7)$. Thus the pooled group of PT trainees included 242 trainees. $^{1}$

\footnotetext{
${ }^{1}$ In the statistical analyses, we found only minimal differences between psychodynamic psychotherapy and psychoanalyses regarding topics and techniques in supervision. Regarding topics, 'therapist competencies' (item 14) was significantly more often addressed in psychoanalyses-oriented supervisions. Regarding techniques, 'agenda setting' (item 4) was more often used in psychodynamic-oriented supervisions and 'therapist cognitive processes' were more often used in psychoanalyses-oriented supervisions. However, the effect sizes were only of small magnitude.
} 
Table 1. Characteristics of the participants $(n=791)$

\begin{tabular}{ll}
\hline Characteristic & \\
\hline Female (\%) & $666(84.2)$ \\
Age $(S D)$ & $32.9(6.6)$ \\
Professional group & \\
$\quad$ Psychologist (\%) & $637(80.5)$ \\
Pedagogue (\%) & $135(17.1)$ \\
Other & $19(2.4)$ \\
Psychotherapeutic orientation & \\
Cognitive behavioural (\%) & $533(67.4)$ \\
Psychodynamic (\%) & $163(20.6)$ \\
Psychoanalysis (\%) & $72(9.1)$ \\
Cognitive behavioural and psychodynamic (\%) & $9(1.1)$ \\
Psychodynamic and psychoanalysis & $7(0.9)$ \\
Other (i.e. systemic, gestalt, humanistic, eclectic) & $7(0.9)$ \\
Primary treatment group & \\
Adults (\%) & $597(75.5)$ \\
Children/youths (\%) & $179(22.6)$ \\
Treated both groups equally (\%) & $15(1.9)$ \\
Years since the beginning of psychotherapy training $(S D)$ & $3.4(2.2)$ \\
Number of supervision sessions thus far (SD) & $68.5(70.1)$ \\
Number of therapy sessions conducted in a week (SD) & $10.7(6.7)$ \\
\hline
\end{tabular}

\section{Participants}

A total of 791 participants fulfilled our inclusion criteria and were considered for the data analysis (533 CBT and 242 PT). The characteristics of the participants are given in Table 1. Note that a pedagogue is an individual who has studied pedagogy (methods of teaching) at a university. We found significant differences between trainees of different therapeutic orientations. Trainees of PT were an average of 4.6 years older $(F(1,773)=90.24 ; p<.001)$, had received an average of 14.1 more supervision sessions $(F(1,773)=6.64 ; p=.01)$ and had an average of 1.04 years more psychotherapy training $(F(1,773)=40.96 ; p<.001)$ than trainees of CBT. No differences were found regarding $\operatorname{sex}\left(\chi^{2}(1)=1.76 ; p=.20\right)$ between trainees of PT and CBT.

\section{Survey questionnaire}

The survey questionnaire of the current study was based on a previous survey on clinical supervision (Townend et al., 2002) and included four sections. The first section assessed sociodemographic data (i.e. sex, age, university education, and psychotherapeutic orientation).

The second section assessed details regarding psychotherapy training, psychotherapeutic activities, and clinical supervision (i.e. start of psychotherapy training, whether psychotherapy training was finished, number of weekly therapy sessions, number of different supervisors, and number of received supervisory sessions).

The third section assessed the frequency of 16 topics in clinical supervision: (1) the therapeutic alliance, (2) therapeutic goals and agreements, (3) patient motivation and 
suitability, (4) maintaining factors, (5) case reports regarding symptoms and progress, (6) treatment plan, (7) selection and implementation of interventions, (8) patient homework, (9) evaluation of the treatment or the treatment progress, (10) ethical issues, (11) risk evaluation regarding the patient (e.g. suicidality) or evaluation of risks for the therapist due to the patient, (12) diagnostic issues, (13) therapy success, (14) therapist competencies, (15) therapist's own personal problems, and (16) satisfaction with the supervision. Supervisees were asked how often those topics were part of their supervision sessions. The response format for the 16 items regarding the supervision topics was a four-point scale $(1=$ never, $2=$ rarely, $3=$ sometimes, and $4=$ often).

The fourth section assessed the frequency of 10 techniques in clinical supervision: (1) direct observation (live supervision), (2) consideration of audiotapes or videotapes, (3) role play, (4) agenda setting for the supervisory session, (5) personal goal setting for the supervisee, (6) case discussion, (7) considering the supervisee's own cognitive processes and their relevance for the therapeutic behaviour, (8) therapist homework tasks, (9) information brokering (e.g. regarding interventions or diagnosis), and (10) recommending literature (e.g. manuals, worksheets, diagnostic instruments). Supervisees were asked how often those techniques were used in their supervision sessions. The response format of the 10 items regarding supervision techniques was a four-point scale $(1=$ never, $2=$ rarely, $3=$ sometimes, and $4=$ often).

The internal consistency (Cronbach's $\alpha$ ) of the 26 items assessing supervisory topics and techniques was .81 . The Cronbach's $\alpha$ subscale for the assessment of topics (16 items) was .76 , and for the subscale for the assessment of techniques (10 items) was .65.

\section{Statistical analyses}

Analyses of variance (ANOVA) were used to compare the dimensional variables. Thereby, CBT and PT were considered group factors and the dimensional variables (e.g. age) were considered dependent variables. Differences between CBT and PT in terms of the categorical variables (e.g. sex) were analysed using chi-squared tests. Differences in the frequency of topics and techniques in clinical supervision between groups (CBT versus PT) were analysed using two multivariate analyses of variance (MANOVA). In the first MANOVA, the 15 items that measured topics were defined as the dependent variables and the supervisory group (CBT and PT) was the independent variable (relevant for hypothesis 1). In the second MANOVA, the 10 items that measured techniques were defined as the dependent variables and the supervisory group (CBT and PT) was the independent variable (relevant for hypothesis 2). Because of the differences in the group sample sizes, between-group effect sizes were determined by Hedges' $g$. A Hedges' $g$ of .40 is considered a small effect, a Hedges' $g$ between .50 and .80 is considered a moderate effect, and a Hedges' $g$ larger than 0.80 is considered a large effect size (Cohen, 1988). Correlations, which were relevant for hypothesis 3 , were analysed using the Pearson correlation coefficient.

\section{Results}

\section{Frequency of specific topics}

Figure 2 and Table 2 present the frequency of different topics in clinical supervision. The most frequently discussed topics were 'interventions' (item 7), 'therapeutic alliance' (item 1), 


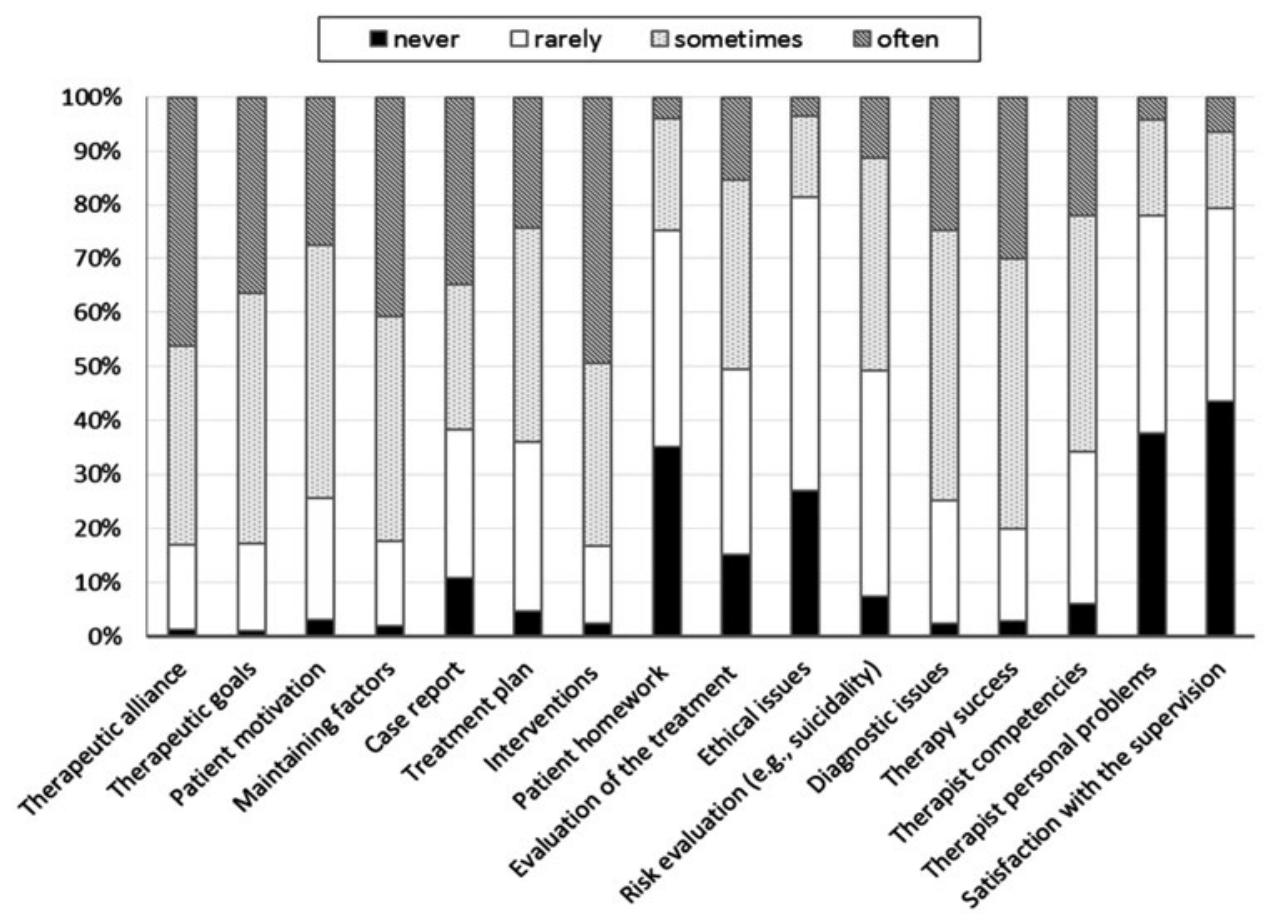

Fig. 2. Frequency of the different topics in clinical supervision of psychotherapy trainees $(n=791)$

'maintaining factors' (item 4), and 'therapeutic goals' (item 2). The less frequently discussed topics were 'satisfaction with the supervision', 'therapist personal problems', and 'ethical issues'.

To address the first hypothesis, we compared the frequency of supervisory topics in CBT and PT. In the MANOVA, significant differences between CBT and PT approaches were found for supervisory topics $(V=0.40 ; F(1,773)=31.30 ; p<.001)$. For the different topics, most effect sizes for between-group differences were of small or moderate magnitude (see Table 2). Only for 'therapeutic alliance' (item 1; $g=-0.88$ ) and 'patient homework' (item $8 ; g=1.24)$ were large effect sizes found. The 'therapeutic alliance' was discussed more frequently in PT-oriented than in CBT-oriented supervisions, and 'patient homework' was discussed more frequently in CBT-oriented than in PT-oriented supervisions. In contrast to the third hypothesis, the frequency of specific topics was not correlated or was only marginally correlated with the number of received supervision sessions or the duration of psychotherapy training (all $r$ values $<.20$ ).

\section{Frequency of techniques used}

Figure 3 and Table 3 present the frequency of different techniques used in supervision. The most frequent technique was 'case discussion' (item 6), followed by 'information brokering' (item 9). Several techniques were seldom used, such as 'direct observation' (item 1), 'therapist homework tasks' (item 8), 'role play’ (item 3), and 'audio/videotapes' (item 2). 
Table 2. Mean scores and standard deviations regarding specific topics in supervision

\begin{tabular}{lllc}
\hline & $\begin{array}{l}\text { Cognitive } \\
\text { behavioural } \\
\text { therapy* } \\
(n=533)\end{array}$ & $\begin{array}{l}\text { Psychodynamic } \\
\text { therapy* } \\
(n=242)\end{array}$ & $\begin{array}{l}\text { Effect sizes } \\
\text { of group } \\
\text { differences } \\
\text { Hedge's } g\end{array}$ \\
\hline 7. Interventions & $3.38(0.77)$ & $3.14(0.85)$ & 0.30 \\
2. Therapeutic goals & $3.25(0.73)$ & $3.01(0.74)$ & 0.33 \\
4. Maintaining factors & $3.25(0.76)$ & $3.11(0.81)$ & 0.18 \\
1. Therapeutic alliance & $3.08(0.78)$ & $3.71(0.56)$ & -0.88 \\
13. Therapy success & $3.06(0.79)$ & $3.12(0.69)$ & $0.08^{\text {n.s. }}$ \\
12. Diagnostic issues & $3.02(0.76)$ & $2.86(0.72)$ & 0.21 \\
3. Patient motivation & $2.98(0.78)$ & $2.98(0.80)$ & $0.00^{\text {n.s. }}$ \\
6. Treatment plan & $2.90(0.84)$ & $2.68(0.85)$ & 0.26 \\
5. Case report & $2.77(1.03)$ & $3.02(0.97)$ & -0.25 \\
14. Therapist competencies & $2.70(0.84)$ & $3.07(0.79)$ & -0.45 \\
9. Evaluation of the treatment & $2.50(0.92)$ & $2.52(0.95)$ & $0.02^{\text {n.s. }}$ \\
11. Risk evaluation & $2.49(0.79)$ & $2.66(0.76)$ & -0.22 \\
8. Patient homework & $2.22(0.81)$ & $1.31(0.54)$ & 1.24 \\
10. Ethical issues & $1.84(0.69)$ & $2.20(0.80)$ & -0.50 \\
15. Therapist personal problems & $1.80(0.81)$ & $2.08(0.88)$ & -0.34 \\
16. Satisfaction with the supervision & $1.74(0.87)$ & $2.05(0.93)$ & -0.35 \\
\hline
\end{tabular}

Items were ordered according to their frequency in cognitive behavioural supervisions, beginning with the most frequent topic. ${ }^{*}$ Scale range: $1=$ never, $2=$ rarely, $3=$ sometimes, $4=$ often; n.s. $=$ not significant.

To address the second hypothesis, we compared the frequency of supervisory topics in CBT and PT. Supervision in CBT and PT approaches differed significantly in the MANOVA for supervisory techniques $(V=0.31 ; F(1,773)=34.08 ; p<.001)$. None of the effect sizes for the between-group differences was of large magnitude. The largest effect sizes were found for the use of 'audio/videotapes' (item 2; $g=0.69$ ) and for 'agenda setting' (item $4 ; g=$ 0.64). 'Audio/videotapes' were used more frequently in CBT-oriented than in PT-oriented supervisions, and an agenda was implemented more often in CBT-oriented than in PT-oriented supervisions. In contrast to the third hypothesis, the frequency of specific techniques was not correlated or was only marginally correlated with the number of received supervision sessions or the duration of psychotherapy training (all $r$ values $<.20$ ).

\section{Discussion}

The current study investigated topics and techniques in clinical supervision in a large sample of psychotherapy trainees in Germany. The findings showed that supervision focused on topics that are central for the effective treatment of patients (i.e. therapeutic interventions, therapeutic alliance, maintaining factors, therapeutic goals). However, we found that active strategies (e.g. role play), structuring strategies (e.g. agenda setting), and strategies that allow for feedback regarding supervisees' competencies (e.g. via the use of audiotapes or videotapes) were seldom used in clinical supervision. Case discussion was the predominant 
Table 3. Mean scores and standard deviations regarding specific techniques in supervision

\begin{tabular}{lllc}
\hline & $\begin{array}{l}\text { Cognitive- } \\
\text { behavioral } \\
\text { therapy* } \\
(n=533)\end{array}$ & $\begin{array}{l}\text { Psychodynamic } \\
\text { therapy* } \\
(n=242)\end{array}$ & $\begin{array}{l}\text { Effect sizes } \\
\text { of group } \\
\text { differences } \\
\text { Hedge's } g\end{array}$ \\
\hline 6. Case discussion & $3.42(0.84)$ & $3.57(0.73)$ & -0.19 \\
9. Information brokering & $3.07(0.88)$ & $2.89(0.91)$ & 0.20 \\
10. Recommending literature & $2.86(0.95)$ & $2.14(0.91)$ & 0.77 \\
7. Therapist cognitive processes & $2.47(0.89)$ & $2.97(0.98)$ & -0.54 \\
2. Audio/videotapes & $2.20(1.04)$ & $1.50(0.97)$ & 0.69 \\
4. Agenda setting & $2.17(1.14)$ & $1.49(0.84)$ & 0.64 \\
5. Personal goal setting & $1.88(0.95)$ & $1.64(0.81)$ & 0.26 \\
3. Role play & $1.72(0.83)$ & $1.34(0.66)$ & 0.49 \\
8. Therapist homework tasks & $1.48(0.67)$ & $1.38(0.67)$ & $0.15^{\text {n.s. }}$ \\
1. Direct observation & $1.14(0.48)$ & $1.10(0.39)$ & $0.09^{\text {n.s. }}$ \\
\hline
\end{tabular}

Items were ordered according to their frequency in cognitive behavioural supervisions, beginning with the most frequent technique. ${ }^{*}$ Scale range: $1=$ never, $2=$ rarely, $3=$ sometimes, $4=$ often; n.s. $=$ not significant.

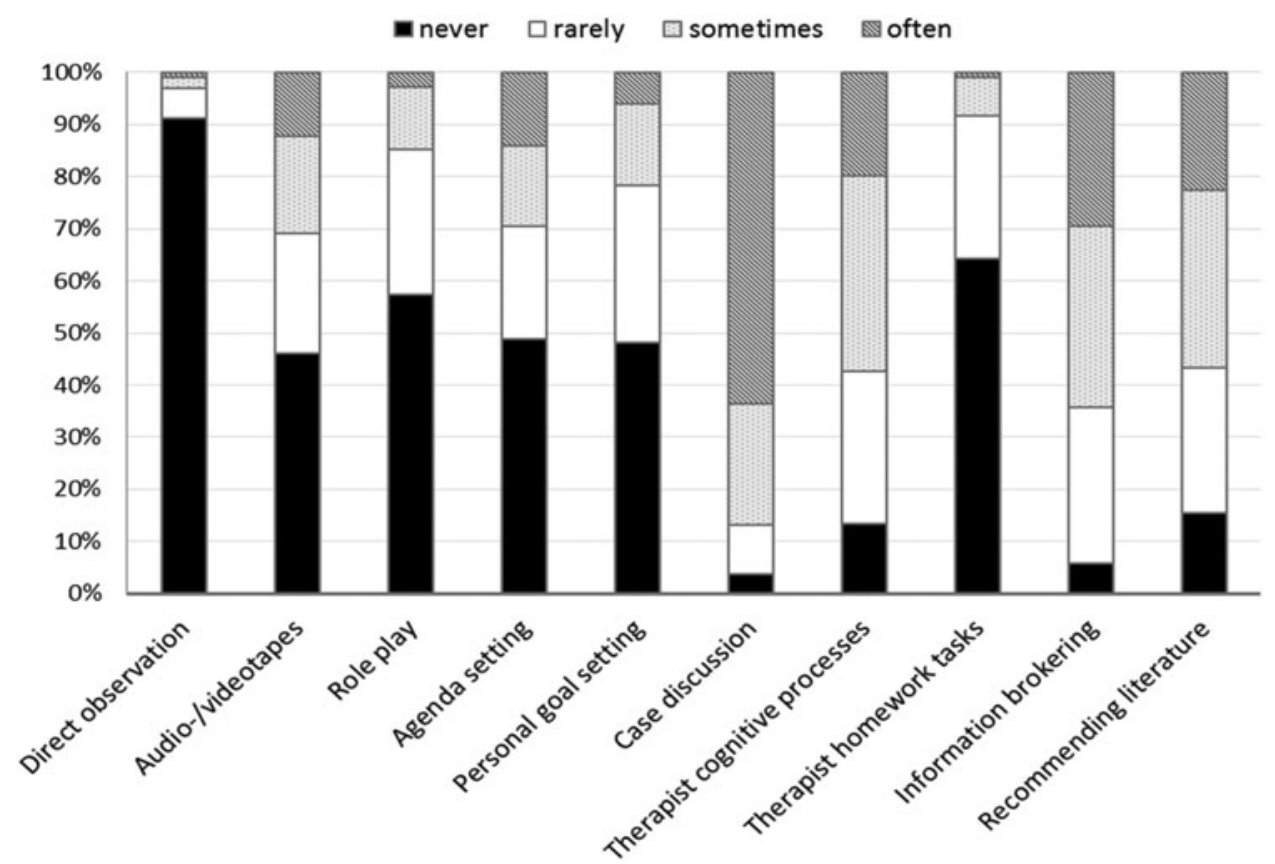

Fig. 3. Frequency of the different techniques in clinical supervision of psychotherapy trainees $(n=791)$ 
strategy in clinical supervision of psychotherapy trainees. In contrast to hypotheses 1 and 2, we have found rather small differences between CBT-oriented and PT-oriented supervisions. In contrast to hypothesis 3, we found that supervisees' characteristics are less relevant for the reported supervisory topics and techniques.

Overall, our results are comparable to the findings of Townend et al. (2002) who investigated accredited psychotherapists in the UK. Clinical supervision was less active and less structured than recommended in the literature. For example, $46 \%$ of the supervisees reported that they never used audiotapes or videotapes. This phenomenon might make clinical supervision less effective than it could otherwise be. Empirical research has demonstrated that therapists have difficulty adequately evaluating their competencies and that video-based or observation-based feedback was superior for improving therapeutic competencies. One reason for this discrepancy between recommendations and clinical practice might be that direct observation is associated with fears of negative evaluation by the supervisor. These fears are related to non-disclosure (Jakob et al., 2014; Sweeney and Creaner, 2014), which is easier to realize within case discussions than within strategies based on direct observation. Therefore, supervisees may avoid more direct supervision strategies (e.g. use of videotapes, direct observation). Another reason might be that strategies that are more direct are also more time consuming. For example, live supervision, which allows for direct observation of therapeutic competencies and direct feedback, is associated with larger organizational costs (Jakob et al., 2015). Furthermore, there might be a lack of knowledge regarding the appropriate implementation of more active or direct strategies, such as videotapes, in supervision sessions. Thus supervisors and supervisees may seldom use these techniques. Townend et al. (2002) described this issue critically more than 15 years ago as follows: 'What this means is that supervision is mainly about what supervisees say they are doing in practice and not what may actually be taking place' (p. 497). In contrast to the study by Townend et al. (2002), we did not investigate accredited psychotherapists but psychotherapy trainees. In that context, the lack of active strategies can be evaluated as even more problematic (O'Donovan et al., 2011).

The current study and the study by Townend et al. (2002) differ from more recent studies, which found that active and structuring strategies were used more frequently (Reiser and Milne, 2016; Gonsalvez et al., 2017). However, those more recent studies also differed significantly regarding the frequency of specific supervisory methods. In the study by Gonsalvez et al. (2017), 46\% of the supervisors reported reviewing audio/videotapes 'never' or 'rarely', whereas in the study by Reiser and Milne (2016), only $1 \%$ of the supervisors reported reviewing audio/videotapes 'never' or 'rarely'. Differences between the current study and the more recent studies might be due to different perspectives because the more recent studies surveyed supervisors and not supervisees. It is possible that the information given by supervisors and supervisees is incongruent. A further explanation might be that supervisory practices in the UK have changed and that supervisory practices in Germany are comparable to those in the UK 15 years ago. Therefore, future studies should include both the perspective of the supervisors and the supervisees. In addition, observational studies would allow a more objective evaluation of supervisory practices.

In addition to the study by Townend et al. (2002), CBT as well as PT trainees were considered in the current study. In contrast to our hypotheses 1 and 2, we found that differences between CBT and PT regarding topics and techniques in clinical supervision were not present, or were mostly of small to moderate magnitude. This result is surprising 
because theoretical models of supervision would suggest a different approach in CBT and PT supervisions. One explanation for this finding might be that the recommendations regarding CBT supervision (i.e. structured and active) are not implemented satisfactorily. For example, in our study, case discussion was the dominant technique in CBT and PT and more active techniques (e.g. role play), which are particularly preferred in CBT, were seldom used. Because of this lack of specificity in CBT supervision, differences between CBT and PT might be marginal for most areas.

Strong differences between CBT-oriented and PT-oriented supervisions were only found for the frequency of addressing the therapeutic alliance $(g=-0.88)$ and patient homework $(g$ $=1.24)$. This partial result is in line with our first hypothesis, but in contrast with other topics (e.g. interventions), where the differences between CBT and PT were only marginal. Homework tasks are not confined to one therapeutic orientation but are particularly relevant in CBT (Kazantzis and Dattilio, 2010). Therefore, it is in accordance with theoretical models stating that homework tasks are more often a topic in CBT-oriented supervisions. The therapeutic alliance, in contrast, is of primary importance in PT, but is also considered an important therapeutic process in CBT and is significantly related to therapy success (Horvath et al., 2011). Therefore, it might be an important difference that the therapeutic alliance is more frequently a topic in PT than in CBT. The empirical findings suggest that the quality of the therapeutic alliance in CBT is as good as in PT (e.g. Raue et al., 1995). However, we cannot rule out the possibility that those differences influence the effectiveness of the given supervision.

For some techniques, we found moderate differences between CBT and PT. We found that PT-oriented supervisions were less structured (e.g. agenda setting, personal goal setting) than CBT-oriented supervisions. This partial result is also in line with hypotheses 2 because structure is seen as more relevant in CBT than in psychodynamic treatments (Cutler et al., 2004). However, the fact that we seldom found the use of structuring strategies in CBToriented supervisions contradicts recommendations regarding the effective design of clinical supervision in CBT (Cummings et al., 2015).

In terms of our third hypothesis, we found no evidence that different supervision strategies were used in relation to the training progress of the trainees. This result is surprising when we consider the development models for psychotherapy competencies (Milne et al., 2001; Sharpless and Barber, 2009) because these models imply that trainees have different learning needs in relation to their level of competence. In particular, in psychotherapy training, a stronger adapted supervisory practice would be desirable.

\section{Limitations}

Our assessment method was limited to supervisees' retrospective reports and is potentially confounded by memory biases. Therefore, direct observation of supervision sessions would be preferable. Our recruiting method (due to the postgraduate psychotherapy training institutions) did not allow us to determine how many psychotherapy trainees received the link to our study; we can only determine how many participants started the survey. For this sample, our response rate (60\% included in the data analysis) was comparable to the survey of Townend et al. (2002), which had a response rate of $61 \%$, and was larger than that of the study by Reiser and Milne (2016; response rate: 44\%) and the study by Gonsalvez et al. (2017; response rate: $18 \%$ ). The response rate in our study can be seen as very high when we consider that $91 \%$ of the participants who started the survey completed the sociodemographic 
data section. Moreover, the assessment was conducted via the internet, which might influence participants' responses. However, empirical findings show that internet findings are consistent with findings from traditional assessment methods (Gosling et al., 2004).

Furthermore, while the reliability of the whole measure (Cronbach's $\alpha=.81$ ) and the subscale supervisory topics (Cronbach's $\alpha=.76$ ) were satisfactory, the reliability of the subscale supervisory techniques (Cronbach's $\alpha=.65$ ) was questionable. One explanation for the low reliability of the subscale supervisory techniques might be that the scale assessed relatively heterogeneous supervisory techniques. Thus the intercorrelations of the items and consequently the internal consistency of the scale were low. Therefore, the test-retest reliability might be a better estimator of the reliability; however, test-retest reliability was not evaluated in the current study. The study by Reiser and Milne (2016) reported the reliability of the measurement, but reliability was not reported by the Townend et al. (2002) or Gonsalvez et al. (2017) studies. In the study by Reiser and Milne (2016), the internal consistencies of the measures were satisfactory. However, a very homogenous sample of supervisors was interviewed, which might explain the more homogenous use of supervisory methods and the higher internal consistency of the measurement.

Another limitation is that supervisees did not differ only with regard to their therapeutic orientations. Trainees of PT orientations were older, had received more supervision sessions and had been in psychotherapy training longer than trainees of CBT. These characteristics might have influenced the frequency of topics and techniques in clinical supervision. However, in our analyses, we found no correlations or only small correlations between those characteristics and the frequency of topics and techniques in clinical supervision. Therefore, we can assume that the differences are attributable to the therapeutic orientations rather than to those characteristics.

Furthermore, the current study was limited to clinical supervision in CBT and PT. These therapeutic approaches are the most common and important in Germany. However, other therapeutic approaches, such as interpersonal psychotherapy or systemic therapy, are also relevant and should be investigated in future studies. Clinical supervision in additional countries should also be considered.

In addition to the Likert scales used in the current study, qualitative data regarding clinical supervision would also be interesting (see Townend et al., 2002). For example, supervisees could be questioned about the topics and techniques they identified during the supervision sessions. It would be interesting to determine whether the supervisees would name additional topics and techniques. Therefore, further studies should also consider open questions and qualitative analyses in supervision research.

\section{Conclusions}

Consistent with findings from more than a decade ago in the UK (Townend et al., 2002) and in contrast to more recent studies (e.g. Reiser and Milne, 2016), the current study shows that supervision practices in psychotherapy training differ substantially from experts' recommendations and empirical evidence. Clinical supervision is a training element that can go beyond other training elements (e.g. workshops) by providing direct feedback regarding trainees' competencies. In line with this consideration, directors of psychotherapy training institutes see supervision as the most appropriate part of training to identify deficits in trainees' competencies (Nodop and Strauß, 2013). However, this potential of clinical 
supervision is currently not fully used in clinical practice. To date in Germany, only practical experience as a psychotherapist and trainer are required to become a supervisor in psychotherapy training. Thus it can be questioned whether this procedure is sufficient. In contrast, it could be argued that a professional and state-approved supervisory training programme that considers current recommendations for supervisory practice is necessary.

\section{Main points}

(1) Literature recommendations and empirical research on clinical supervision emphasize the importance of providing accurate feedback (e.g. by using videotapes) to ensure effective supervision.

(2) Within the context of psychotherapy training, we found that clinical supervision seldom uses active strategies, structuring strategies or strategies that allow for direct feedback regarding supervisees' competencies (e.g. the use of audiotapes or videotapes).

(3) Differences regarding topics and techniques in clinical supervision between different therapeutic orientations (cognitive behavioural therapy and psychodynamic psychotherapy) were only marginal.

(4) To ensure supervisees' optimal learning, methods that allow the direct evaluation of supervisees' competencies more should be considered (e.g. live supervisions or the use of videotapes).

\section{Ethical statements}

The authors assert that all procedures that contributed to this work comply with the Ethical Principles of Psychologists and Code of Conduct as set out by the APA. Because this study was considered a low-risk study and did not include patients, invasive procedures, or deception, our institution does not require official approval by an ethics committee.

\section{Conflicts of interest}

Florian Weck, Yvonne M. Kaufmann and Michael Witthöft have no conflicts of interest with respect to this publication.

\section{Recommended follow-up reading}

Muse K, McManus F (2013). A systematic review of methods for assessing competence in cognitivebehavioural therapy. Clinical Psychology Review 33, 484-499.

Rakovshik SG, McManus F (2010). Establishing evidence-based training in cognitive behavioral therapy: a review of current empirical findings and theoretical guidance. Clinical Psychology Review 30, 496-516.

\section{References}

Bambling M (2014). Creating positive outcomes in clinical supervision. In The Wiley International Handbook of Clinical Supervision (eds Watkins CE, Milne DL), pp. 445-457. Chichester: WileyBlackwell. 
Carmel A, Villatte JL, Rosenthal MZ, Chalker S, Comtois KA (2016). Applying technological approaches to clinical supervision in dialectical behavior therapy: a randomized feasibility trial of the bug-in-the-eye (BITE) model. Cognitive and Behavioral Practice 23, 221-229.

Cohen J (1988). Statistical Power Analysis for the Behavioral Sciences. Hillsdale, NJ: Erlbaum.

Cummings JA, Ballantyne EC, Scallion LM (2015). Essential processes for cognitive behavioral clinical supervision: agenda setting, problem-solving, and formative feedback. Psychotherapy 52, $158-163$.

Cutler JL, Goldyne A, Markowitz JC, Devlin MJ, Glick RA (2004). Comparing cognitive behavior therapy, interpersonal psychotherapy, and psychodynamic psychotherapy. American Journal of Psychiatry 161, 1567-1573.

Falender CA, Shafranske EP (2014). Clinical supervision: the state of the art. Journal of Clinical Psychology 70, 1030-1041.

Gonsalvez CJ, Wahnon T, Deane FP (2017). Goal-setting, feedback, and assessment practices reported by Australian clinical supervisors. Australian Psychologist 52, 21-30.

Gosling SD, Vazire S, Srivastava S, John OP (2004). Should we trust web-based studies? A comparative analysis of six preconceptions about Internet questionnaires. American Psychologist 59, 93-104.

Horvath AO, Del Re AC, Flückiger C, Symonds D (2011). Alliance in individual psychotherapy. Psychotherapy 48, 9-16.

Jakob M, Weck F, Höfling V, Richtberg S, Bohus M (2014). Non-disclosure during psychotherapy supervision: validation of the German version of the supervisory questionnaire (SQ). Psychotherapy Research 24, 42-51.

Jakob M, Weck F, Schornick M, Krause T, Bohus M (2015). Wenn der Supervisor zuschaut. Eine qualitative Analyse der Akzeptanz von Live-Supervision. [When the supervisor is watching. Qualitative analysis of the acceptance of live supervision]. Psychotherapeut 60, 210-215.

Kazantzis N, Dattilio FM (2010). Definitions of homework, types of homework, and ratings of the importance of homework among psychologists with cognitive behavior therapy and psychoanalytic theoretical orientations. Journal of Clinical Psychology 66, 758-773.

Lambert MJ, Ogles BM (1997). The effectiveness of psychotherapy supervision. In Handbook of Psychotherapy Supervision (ed Watkins CE), pp. 421-446. New York: Wiley and Sons.

Martino S, Ball S, Nich C, Frankforter TL, Carroll KM (2009). Correspondence of motivational enhancement treatment integrity ratings among therapists, supervisors, and observers. Psychotherapy Research 19, 181-193.

Miller WR, Yahne CE, Moyers TB, Martinez J, Pirritano M (2004). A randomized trial of methods to help clinicians learn motivational interviewing. Journal of Consulting and Clinical Psychology $\mathbf{7 2}$, 1050-1062.

Milne D (2007). An empirical definition of clinical supervision. British Journal of Clinical Psychology 46, 437-447.

Milne D, Claydon T, Blackburn I-M, James I, Sheikh A (2001). Rationale for a new measure of competence in therapy. Behavioural and Cognitive Psychotherapy 29, 21-33.

Milne D, Reiser R, Aylott H, Dunkerley C, Fitzpatrick H, Wharton S (2010). The systematic review as an empirical approach to improving CBT supervision. International Journal of Cognitive Therapy 3, 278-294.

Nodop S, Strauß B (2013). Mangelnde Eignung bei angehenden Psychotherapeuten. Psychotherapeut 58, 446-454.

Nodop S, Thiel K, Strauß B (2010). Supervision in der psychotherapeutischen Ausbildung in Deutschland. Psychotherapeut 55, 485-495.

O'Donovan A, Halford WK, Walters B (2011). Towards best practice supervision of clinical psychology trainees. Australian Psychologist 46, 101-112. 
Rakovshik SG, McManus F (2010). Establishing evidence-based training in cognitive behavioral therapy: a review of current empirical findings and theoretical guidance. Clinical Psychology Review 30, 496-516.

Raue P, Putterman J, Goldfried M, Wolitzky D (1995). Effect of rater orientation on the evaluation of therapeutic alliance. Psychotherapy Research 5, 337-342.

Reiser RP, Milne DL (2016). A survey of CBT supervision in the UK: methods, satisfaction and training, as viewed by a selected sample of CBT supervision leaders. the Cogntive Behaviour Therapist 9, e20. doi: 10.1017/S1754470X15000689

Sharpless BA, Barber JP (2009). A conceptual and empirical review of the meaning, measurement, development, and teaching of intervention competence in clinical psychology. Clinical Psychology Review 29, 47-56.

Smith JL, Carpenter KM, Amrhein PC, Brooks AC, Levin D, Schreiber EA, Travaglini LA, Hu MC, Nunes EV (2012). Training substance abuse clinicians in motivational interviewing using live supervision via teleconferencing. Journal of Consulting and Clinical Psychology 80, 450-464.

Strauß B, Kohl S (2009). Entwicklung der Psychotherapie und der Psychotherapieausbildung in europäischen Ländern. Psychotherapeut 54, 457-463.

Strauß B, Wheeler S, Nodop S (2010). Klinische Supervision. Überblick über den Stand der Forschung [Clinical supervison. Review of the state of research]. Psychotherapeut 55, 455-464.

Sweeney J, Creaner M (2014). What's not being said? Recollections of non-disclosure in clinical supervision while in training. British Journal of Guidance and Counselling 42, 211-224.

Townend M, Iannetta L, Freeston MH (2002). Clinical supervision in practice: a survey of UK cognitive behavioural psychotherapists accredited by the BABCP. Behavioural and Cognitive Psychotherapy 30, 485-500.

Walfish S, McAlister B, O'Donnell P, Lambert MJ (2012). An investigation of self-assessment bias in mental health providers. Psychological Reports 110, 639-644.

Weck F (2013). Psychotherapeutische Kompetenzen: Theorien, Erfassung und Förderung. [Psychotherapy competencies: theory, assessment, and training]. Heidelberg: Springer.

Weck F, Jakob M, Neng JMB, Höfling V, Grikscheit F, Bohus M (2016) The effects of bug-in-theeye supervision on therapeutic alliance and therapist competence in cognitive-behavioral therapy: a randomized controlled trial. Clinical Psychology and Psychotherapy 23, 386-396.

Weck F, Kaufmann YM, Höfling V (2017). Competence feedback improves CBT competence in trainee therapists: a randomized controlled pilot study. Psychotherapy Research 27, 501-509.

Weck F, Richtberg S, Jakob M, Neng JM, Höfling V (2015). Therapist competence and therapeutic alliance are important in the treatment of health anxiety (hypochondriasis). Psychiatry Research 228, 53-58.

Wheeler S, Richards K (2007). The impact of clinical supervision on counsellors and therapists, their practice and their clients. A systematic review of the literature. Counselling and Psychotherapy Research 7, 54-65. 


\section{Learning objectives}

(1) Accurate and direct feedback is important in clinical supervision to ensure optimal learning for psychotherapy trainees.

(2) There is a large discrepancy between recommendations regarding clinical supervision and supervisory practice in psychotherapy training.

(3) Differences in clinical supervision between cognitive behavioural therapy and psychodynamic therapy are rather small in psychotherapy training.

(4) Supervision strategies that address competencies demonstrated by the supervisees (e.g. review of videotapes) should be used more frequently in psychotherapy training. 